\title{
Power Generation on a Bare Electrodynamic Tether during Debris Mitigation in Space
}

\author{
Kan Xie $\mathbb{D}^{1},{ }^{1}$ Fuwen Liang, ${ }^{1}$ Qimeng Xia, ${ }^{1}$ Ningfei Wang, ${ }^{1}$ Zun Zhang, ${ }^{2}$ Haoxiang Yuan, \\ Xiangyang Liu, ${ }^{1}$ and Zhiwen $\mathrm{Wu}^{1}$ \\ ${ }^{1}$ School of Aerospace Engineering, Beijing Institute of Technology, 100081, China \\ ${ }^{2}$ School of Space and Environment, Beihang University, 100191, China \\ Correspondence should be addressed to Kan Xie; xiekan@bit.edu.cn
}

Received 22 July 2020; Revised 22 December 2020; Accepted 3 February 2021; Published 23 February 2021

Academic Editor: Enrico C. Lorenzini

Copyright ( 2021 Kan Xie et al. This is an open access article distributed under the Creative Commons Attribution License, which permits unrestricted use, distribution, and reproduction in any medium, provided the original work is properly cited.

\begin{abstract}
Power generation can be realized in space when current is induced on a bare electrodynamic tether system. The performance of power generation is discussed based on a debris mitigation mission by numerical simulation in the paper. A Li-ion battery subsystem is used to complete the energy conversion-harvest and supply the energy. The battery can provide 10-300 W average electric power continuously during several hundred hour mission time. The energy conversion efficiency ranges from $1 \%$ to a maximum value $30 \%$. With constant power consumption on board, the battery operation generally experiences a discharging phase, a charging phase, and a stable phase. The first two phases determine the mission risk coefficient. The heating problem in the stable phase cannot be ignored. The optimization of battery design and tether design should be considered for each debris mitigation mission. An extra control circuit or small battery voltage with large capacity for battery design is suggested to eliminate the stable phase. Wide or long tether designs are more appropriate for mission with high or low power demands on board, respectively. The power generation is affected by the system mass and the mission orbit parameters.
\end{abstract}

\section{Introduction}

The bare electrodynamic tether is a promising device with which to expand the range of a space mission [1]. It utilizes a bare conductive tether and an electron emission device to complete the charge exchange with the space plasma. The concept of "bare" was first introduced by Sanmartin et al. [2] to avoid the problem of electron collection limitations. The bare conductive tether is used as the anode to absorb electrons and is much more efficient and easier to implement than previous strategies such as anodic plasma contactors or spherical collectors. As the bare electrodynamic tether enables a large current, it can complete many space missions better in LEO (Low Earth Orbit) with high specific impulse and non-or-less propellant consumption when compared to traditional systems $[3,4]$.

When external energy is applied to the tether to change the direction of induced current, the tether works in thruster mode: the Lorentz force component has the same direction as the tether velocity which can be used for orbit raising and drag compensation [5], but more attention has been paid to the passive mode (or generator mode). The bare electrodynamic tether can produce the induced current and significant drag, leading to two impressive functions: power can be generated on board by a load and the system can transfer to a lower orbit. There has been great interest in the latter since the tether was introduced. Due to the characteristics of simplicity, light weight, and no external energy requirement, it is considered to be a potential active debris removal system for unusable satellites and upper stages in LEO $[6,7]$. Much work has been done to better understand the system design $[8,9]$, electron collection [10], stability problems [11-13], system survivability $[14,15]$, and mission performance $[16,17]$.

Although Sanmartin et al. [2] proposed that a bare electrodynamic tether can generate power as far back as 1993, related research mainly appeared after 2000. With the increasing demand for electric power on board, power 


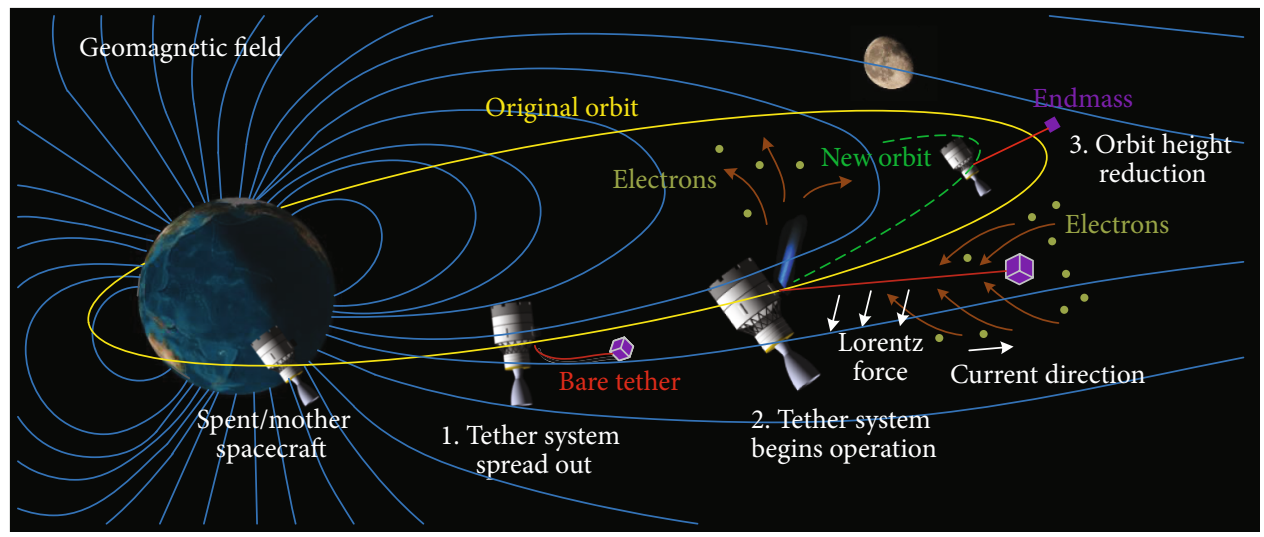

FIGURE 1: Working principle of the bare electrodynamic tether system.

generation by bare electrodynamic tether was gradually taken seriously as the energy can be harvested at any time. Much of the research $[2,18-20]$ focused on the theoretical analysis of power generation performance to estimate the power and efficiency and to suggest the optimization method thereof. The performance of the power generation system will be influenced by the equivalent resistance of each part and the plasma parameters; however, the plasma parameters change during tether operation, so the theoretical formula is usually applicable for point analysis. Numerical simulation with a specific environmental model should be employed to show the long-term performance in terms of power generation. The power and efficiency vary because of the changeable plasma parameters; nevertheless, simulations show that the bare electrodynamic tether was typically efficient for space mission around Jupiter [21] and was a viable device with regard to meeting different power requirements on board [22]. The ProSEDS (Propulsive Small Expendable Deployed System) mission [23] proved that there is no principle problem of power generation from the tether in engineering applications, although the project was later cancelled.

For most published research on energy analysis, there remain some debatable problems: first, the energy storage subsystem model was not accurate, and generally, it was simplified to a constant resistance. This made it convenient to analyse when all parts can be transformed to a uniform parameter, but it is impractical. A normal energy storage subsystem may be thought of in terms of its voltage-current characteristic and maximum capacity. A supercapacitor [24] is more common by contrast. Second, power generation suffers from orbit height reduction which is intolerable for most commercial uses when the tether system is designed merely as a generator. Relevant measures have been proposed by Sanmartin et al. [20] and Hoyt [25] which, however, directly increase system complexity and control difficulty. It is more reasonable to adopt the approach of Sanjurjo-Rivo and Peláez [26] and McTernan et al. [27] who combine the debris mitigation mission and the power generation function. Third, all of the energy analysis was based on maximization of the total cumulative amount; in fact, the energy is harvested and consumed constantly, which means that the energy may not be fully utilized. The dynamic operation of the energy storage subsystem is of practical significance.
Fourth, the influence of tether design and orbit parameters on power generation remains unclear. The equivalent resistance of the energy storage subsystem is usually changed. Theoretical formulae can indeed give estimates under different conditions, but long-term results are unavailable. To make the power generation more practical, these problems cannot be ignored, and they are all considered in the present research.

In this study, the power generation analysis of bare electrodynamic tether is based on a debris mitigation mission. Due to the long mission time (usually weeks or months), a large amount of energy is required to maintain the operation of the electrical appliances on board. Compared with common power sources, this energy source is simple, economical, and sustained. In the numerical simulation, a Li-ion battery system is used as the energy storage subsystem to harvest energy from the tether and provide power for the system at the same time. The battery subsystem is directly connected to the tether. The dynamic behaviour is calculated to assess the working performance in certain situations. Figures and data will show the ability of this power generation system. Furthermore, optimization of the battery design and tether design is discussed. The performance of the system under different mission requirements (mainly system mass and orbit parameters) is also studied.

\section{System Model and Numerical Simulation Settings}

2.1. Physical Process of Power Generation by the Tether System. When the system begins to perform the orbit transfer mission and generate electrical power, the bare tether (of $\mathrm{km}$ in length) is spread out at a low initial velocity from the mother spacecraft (Figure 1). Exploiting the braking system and the gravity gradient, the long, thin, tether finally reaches a steady state in which it is fully straightened, and the vector coincides with the local vertical location. The long conductive tether keeps cutting the geomagnetic induction line at a high speed (typically $8 \mathrm{~km} / \mathrm{s}$ ), creating a considerable induced electromotive force which can be as high as $0.15 \mathrm{~V} / \mathrm{m}$. To produce the induced current, a bare tether and a plasma contactor, both of which will be explained later, are applied. Due to the potential difference resulting from the induced 


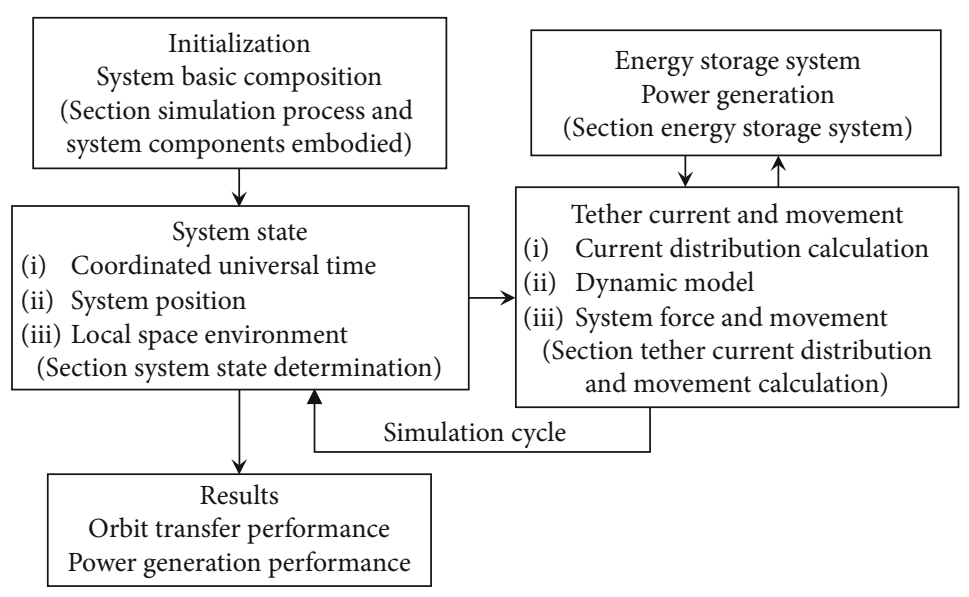

Figure 2: Simulation process.

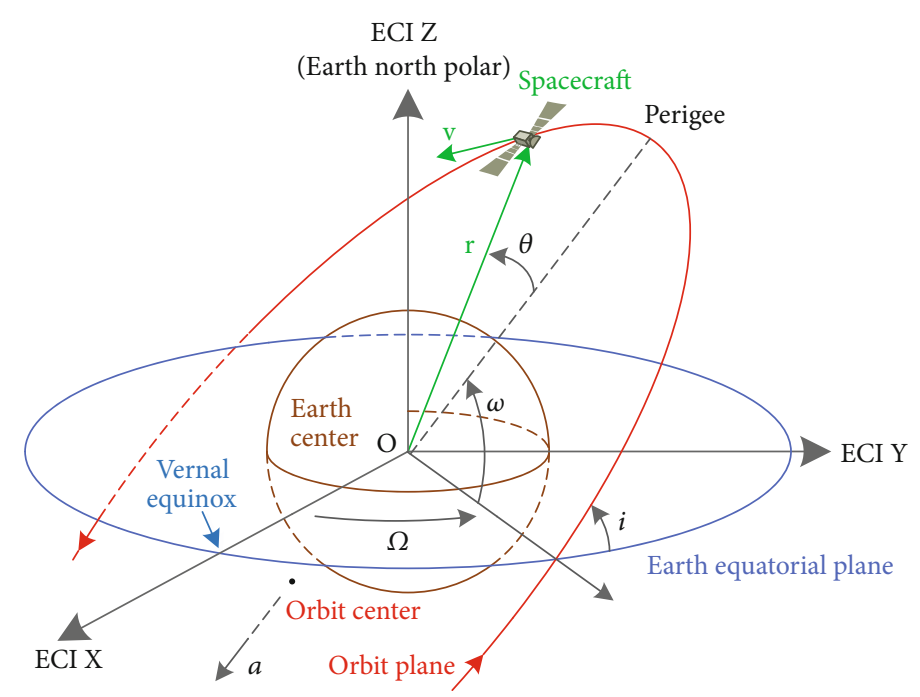

FIGURE 3: ECI coordinate system and classical orbital elements.

electromotive force, the bare tether can collect electrons at a positive potential to the local space plasma potential while the plasma contactor will emit electrons absorbed at a negative potential in a self-consistent manner. Then, power generation is achieved when the induced current flows through the energy storage system. Meanwhile, the Lorenz force whose direction is opposite to the system velocity is created by the current and geomagnetic field, completing the orbit transfer mission.

\subsection{Simulation Process and System Components Embodied.} The simulation process (Figure 2) consists of five parts: initialization, system state determination, tether current distribution and movement calculation, energy storage system operation, and a result. After initialization, the simulation begins to cycle in timed steps. According to the present information pertaining to the tether system, the current and the force on tether can be calculated so that the performance of the power generation can be analysed. Meanwhile, the system updates its location which makes the current in, and force on, the tether changes. The simulation cycle continues until the termination condition is met.
The system is simplified to a combination of a mother spacecraft, a cathodic contactor, a battery system, a bare tether, and an end mass, in turn. The mother spacecraft is a point mass at one end of the tether. The battery system and cathodic contactor are treated as a single massless device but with unique characteristics. The bare tether is regarded as a line with a certain mass distribution and definite shape. The end mass is also a point mass at the other end of the tether. All parts are well connected, both physically and electrically.

2.3. System State Determination. The system state is determined by time, position, and environment. Coordinated Universal Time (UTC) is the time system used to describe orbit propagation. Classical orbital elements in the Earth Centred Inertial (ECI) (Figure 3) coordinate system [28] are employed to furnish detailed information pertaining to the orbit parameters. Six elements can be converted from, and to, the system centre of mass velocity $\mathbf{v}$ and position $\mathbf{r}$, including semimajor axis $a$, eccentricity $e$, inclination $i$, longitude of the ascending node $\Omega$, argument of periapsis $\omega$, and true anomaly $\theta$. The six elements can only be used to describe 


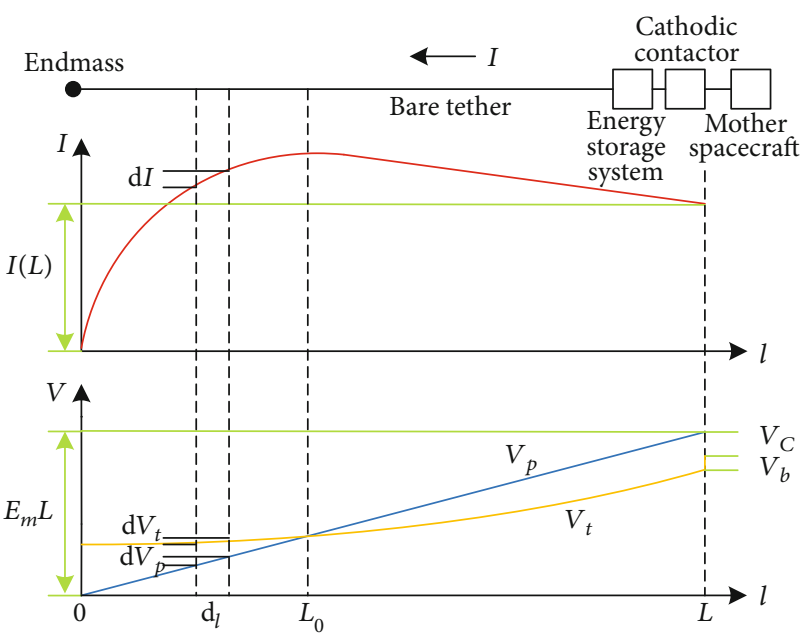

FIgURE 4: Potential and current distribution of a tether system.

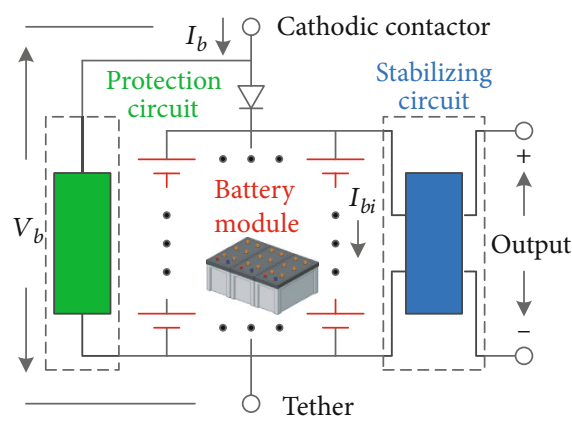

Figure 5: Schematic of the Li-ion battery system.

the movement of the system centre of mass: the velocity and position of other parts in the tether are all inferred therefrom.

The space environment around Earth is complex and changeable. Most parameters vary with time and position. The plasma parameters are obtained from the International Reference Ionosphere (IRI) model [29]. For a given location, time, and date, IRI provides monthly averages of the plasma parameters in the ionospheric altitude range. For example, the plasma density can be as high as $5 \times 10^{11} \mathrm{~m}^{-3}$ in sunshine while as low as $5 \times 10^{9} \mathrm{~m}^{-3}$ in an eclipse at $500 \mathrm{~km}$ height. The geomagnetic field information is provided by the World Magnetic Model (WMM) [30]. From the model, three direction component intensity of the geomagnetic field can be obtained. The total intensity ranges from $2 \times$ $10^{4} \mathrm{nT}$ to $5 \times 10^{4} \mathrm{nT}$. The NRLMSISE-00 model [31] gives the atmosphere total mass density from which the atmospheric drag may be calculated. The air mass density is of the order of $10^{-14}$ or $10^{-13} \mathrm{~kg} \bullet \mathrm{m}^{-3}$ in the given conditions. All the results showed above are at a height of $500 \mathrm{~km}$ and at UTC 2010.01.01.12.00.00.

\subsection{Tether Current Distribution and Movement Calculation.} The current distribution on tether is self-regulated according to the ability of electron collection and emission of the system. Electron collection is realized by the bare tether, and the electron emission is completed by the plasma contactor.
Figure 4 shows the general voltage and current distribution along a bare tether [32]. The tether is so long that it will experience variable conditions in different positions. It can be divided into many short segments (each of length $\mathrm{d} l$ ), each of which has its own potential, current, velocity, and local plasma and geomagnetic field parameters.

For a tether with length $\mathrm{d} l$, the collected current $\mathrm{d} I$ can be described as Equation (1) [2] on the hypothesis of the orbital motion limited theory:

$$
\frac{\mathrm{d} I}{\mathrm{~d} l}= \begin{cases}\frac{\mathrm{e} N_{e} p}{\pi} \sqrt{\frac{2 \mathrm{e}\left(V_{t}-V_{p}\right)}{m_{e}}} & \text { if } V_{t}>V_{p}, \\ 0 & \text { if } V_{t}=V_{p}, \\ -\frac{\mathrm{e} N_{i} p}{\pi} \sqrt{\frac{2 \mathrm{e}\left(V_{p}-V_{t}\right)}{m_{i}}} & \text { if } V_{t}<V_{p} .\end{cases}
$$

The tether potential is caused by the current flowing through the tether resistance while the plasma potential can be regarded as a function of induced electromotive force and is related to the position distribution of the tether [2]:

$$
\begin{aligned}
& \frac{\mathrm{d} V_{t}}{\mathrm{~d} l}=\frac{I}{\sigma A_{t}}, \\
& \frac{\mathrm{d} V_{p}}{\mathrm{~d} l}=E_{m},
\end{aligned}
$$

where $E_{m}$ is the value of vector $\mathbf{E}_{m}=\mathbf{v} \times \mathbf{B}$ at the local tether part. For a certain system position, the distribution of $V_{p}$ is a known quantity. The plasma potential at the end mass point $l=0$ is usually set to be zero so that the $V_{p}$ distribution is obtained:

$$
\begin{aligned}
& V_{p}(0)=0, \\
& V_{p}(L)=\int_{L} E_{m} \mathrm{~d} l .
\end{aligned}
$$

The current at the end mass point $l=0$ and the other end point $l=L$ yield:

$$
\begin{aligned}
& I(0)=0, \\
& I(L)=I_{C} .
\end{aligned}
$$

The plasma contactor is located at the other end point $l=L$ where the electrons are emitted as well as the energy storage system is located before the plasma contactor. The potential distribution at this point is then written as:

$$
V_{t}(L)-V_{p}(L)=-V_{C}-V_{b} .
$$

In the simulation, hollow cathode plasma contactor (HCPC) [33] is employed as the electron emission device. 


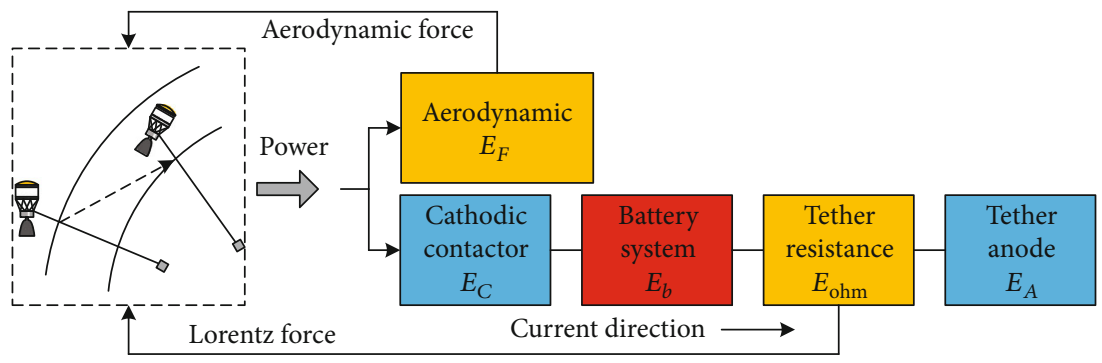

Harvested energy

Drive consumption

Joule heat

Figure 6: Energy distribution in the tether system.

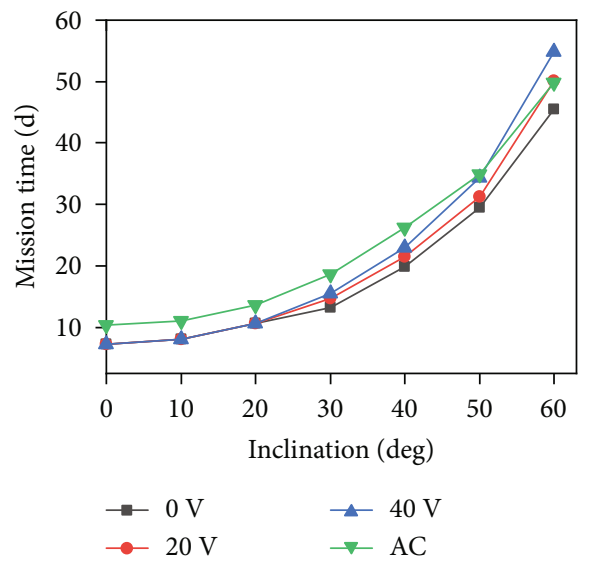

Figure 7: Comparison with the results of deorbiting durations obtained by Sánchez-Arriaga et al. [34].

According to the available data from the experiment conducted in our laboratory, we obtain:

$$
V_{C}= \begin{cases}-172.251 \cdot I_{C}{ }^{2}+176.166 \cdot I_{C} & \text { if } I_{C} \leq 0.5 \mathrm{~A}, \\ 0.069 \cdot I_{C}{ }^{3}-1.027 \cdot I_{C}{ }^{2}+4.890 \cdot I_{C}+42.823 & \text { if } I_{C}>0.5 \mathrm{~A} .\end{cases}
$$

Equations (1)-(7) delineate the current distribution as a nonlinear boundary value problem. The MATLAB ${ }^{\mathrm{TM}}$ inbuilt function "bvp4c" is used to solve the BVP problem by finite iteration. The Lorentz force can be calculated once the current distribution is clear:

$$
\mathrm{dF}_{\mathrm{EDT}}=-\mathbf{B} \times I \mathbf{L d} l .
$$

The force caused by the ambient electric field is not considered as it is usually negligible [24].

The atmospheric drag is:

$$
\mathbf{F}_{\mathrm{air}}=C_{D} \cdot \frac{1}{2} \rho v^{2} \mathbf{S}
$$

where the direction of vector $\mathbf{S}$ is the normal of tether windward side and the value is the equivalent area thereof. The drag coefficient $C_{D}$ is set to 1 .

The whole system is regarded as a centre of mass when describing the movement which means that the libration on the long tether is ignored. The kinetic equation of the system is:

$$
\ddot{\mathbf{r}}=-\frac{\mu}{r^{3}} \mathbf{r}+\frac{\mathbf{F}}{m},
$$

where $\mathbf{F}$ contains all the forces except gravity force. In the simulation, only the Lorentz force $\mathbf{F}_{\mathrm{EDT}}$ and atmospheric drag $\mathbf{F}_{\text {air }}$ are included. Other perturbations are ignored as the mission time is relatively short.

2.5. Energy Storage System. In primary design, a Li-ion battery system is used. The power generation strategy relies on the positive electrode being directly connected to the plasma contactor while the negative one is connected to the bare tether. Figure 5 shows the inner circuit which consists of the battery module, stabilizing circuit, and protection circuit.

The energy storage system can generate and supply electrical power to the battery module and provide energy to maintain on-board electrical appliances. The battery module can also maintain a relatively stable voltage. The protection circuit is used to avoid overcharging of the battery module. For simplification, the voltage of the whole battery system $V_{b}$ is treated as a constant during operation. The efficiency of power generation for the battery module under different and changeable current draw is supposed to be $100 \%$. The performance of the power generation can be represented by:

$$
\begin{aligned}
& P_{b}=V_{b} I_{b}, \\
& E_{b}=\int P_{b} \mathrm{~d} t .
\end{aligned}
$$

The dynamic operation of the battery system is also reviewed. The battery capacity $Q_{\max }$ is set to be $10 \mathrm{~A} \bullet \mathrm{h}$. The working performance under a constant electric consumption on board $P_{o}$ is described by SOC (State of Charge), showed in 
TABLE 1: Basic input parameters.

\begin{tabular}{lcc}
\hline Component & Parameter & Value \\
\hline Mass distribution & Mother spacecraft & $1500 \mathrm{~kg}$ \\
$30 \mathrm{~kg}$ & End mass & $0.5 \mathrm{~cm}$ \\
& $40 \mu \mathrm{m}$ & $10 \mathrm{~km}$ \\
& Cross-sectional length & $2.8 \times 10^{-6} \Omega \bullet \mathrm{cm}^{-3}$ \\
Tether & Cross-sectional width & $2.7 \times 10^{3} \mathrm{~kg} \bullet \mathrm{m}^{-3}$ \\
& Conductive length & $41.9 \mathrm{~V}$ \\
Battery system & Resistivity & $10 \mathrm{~A} \bullet \mathrm{h}$ \\
\hline & Mass density & $6900 \mathrm{~km}$ \\
Orbital parameters & Voltage & $6600 \mathrm{~km}$ \\
& Maximum electrical power & $30^{\circ}$ \\
& Initial semimajor axis & $2010.01 .01,12: 0: 0$ \\
\hline
\end{tabular}

TABle 2: Influence of time step on energy and mission time (normalized to time step $60 \mathrm{~s}$ ).

\begin{tabular}{lcc}
\hline Time step (s) & Energy harvested & Mission time \\
\hline 60 & 1.000 & 1 \\
90 & 0.999 & 0.998 \\
120 & 0.994 & 0.992 \\
\hline
\end{tabular}

Equation (12) and DOD (Depth of Discharge), showed in Equation (13).

$$
\begin{aligned}
\mathrm{SOC} & =\int I_{\mathrm{bi}} \frac{\mathrm{d} t}{Q_{\max }}, \\
\mathrm{DOD} & =1-\mathrm{SOC} .
\end{aligned}
$$

where $I_{\mathrm{bi}}$ is the current flowing through the battery module. When current flows into the battery, it is a positive value; otherwise, it is negative. The battery system has four working modes: (1) if SOC $<1$ and $P_{b}>P_{o}$, tether current flows into the battery module and stabilizing circuit; (2) if SOC $=1$ and $P_{b}>P_{o}$, tether current flows into the protection circuit and stabilizing circuit; (3) if $P_{b}=P_{o}$, tether current flows into the stabilizing circuit only; and (4) if $P_{b}<P_{o}$, extra tether current will flow from the battery module into the stabilizing circuit to make up the power deficit.

2.6. Energy Distribution on the Tether System. The electric power generated arises from the orbital energy of the spacecraft. The orbital energy is the sum of the gravitational potential energy and the kinetic energy of the spacecraft:

$$
E_{\text {orb }}=\frac{-m \mu}{r}+\frac{m v^{2}}{2} \text {. }
$$

This will be constant if there is only gravitational force acting thereon. When the tether system begins its orbit transfer and power generation, the Lorentz force and atmospheric drag lead to an energy change, and the change is equal to the work done thereby:

$$
\Delta E_{\text {orb }}=\int \mathbf{F d} \mathbf{r}=\int \mathbf{F}_{\mathrm{EDT}} \mathrm{d} \mathbf{r}+\int \mathbf{F}_{\mathrm{air}} \mathrm{d} \mathbf{r} .
$$

The orbital energy is then transferred to electrical energy and frictional heating energy, respectively (Figure 6) [24]. Electrical energy is reflected by the current induced on the tether. It is distributed to four parts: the tether anode, tether ohm resistance, battery system, and tether cathode. Energy dissipated on the tether anode $E_{A}$ is used to absorb the ions and electrons while the tether cathode $E_{C}$ is employed to emit electrons. Meanwhile, part of the electrical energy is lost to ohmic Joule heat $E_{\text {ohm }}$. The energy stored by the battery system $E_{b}$ is an energy recovery strategy that can power the electrical appliance. Therefore, the performance of the power generation is mainly determined by the tether current and the equivalent volt-ampere characteristics of other parts. Lastly, a small part of the orbital energy is transferred to frictional heating energy $E_{F}$ caused by aerodynamic drag.

$$
\begin{aligned}
& E_{A}=\int \sum_{n} \Delta V_{t} \cdot \Delta I \mathrm{~d} t, \\
& E_{C}=\int V_{C} \cdot I_{C} \mathrm{~d} t, \\
& E_{\mathrm{ohm}}=\int \sum_{n} \Delta V_{t} \cdot I \mathrm{~d} t, \\
& E_{A}+E_{C}+E_{\mathrm{ohm}}+E_{b}=\int \mathbf{F}_{\mathrm{EDT}} \cdot \mathrm{d} \mathbf{r}, \\
& E_{F}=\int \mathbf{F}_{\mathrm{air}} \cdot \mathrm{d} \mathbf{r} .
\end{aligned}
$$




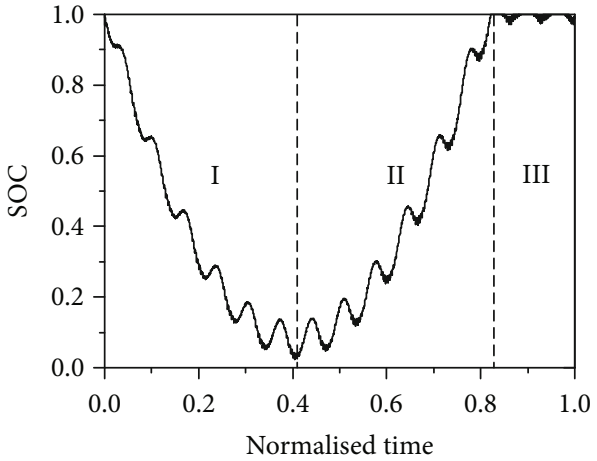

(a)

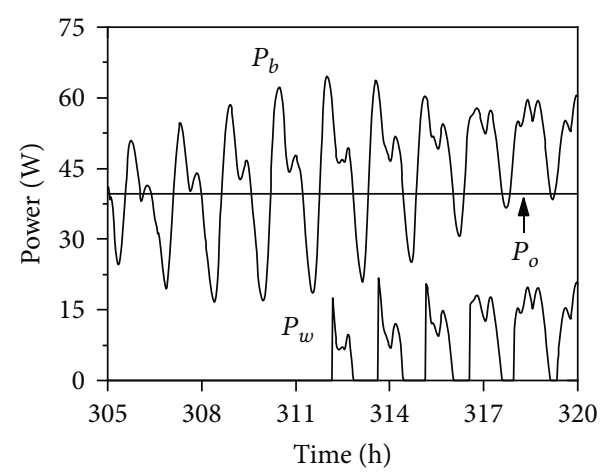

(c)

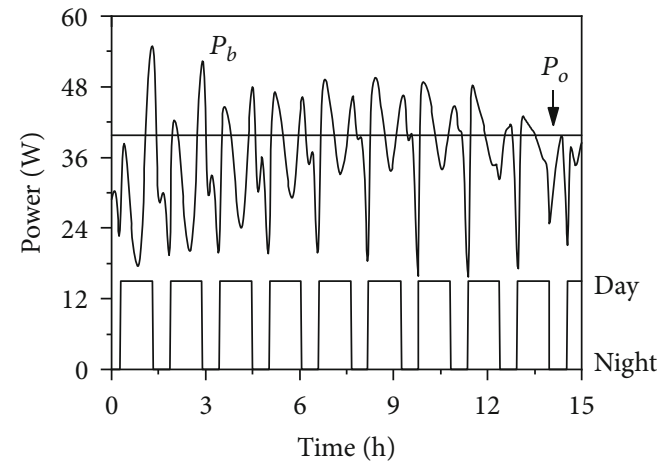

(b)

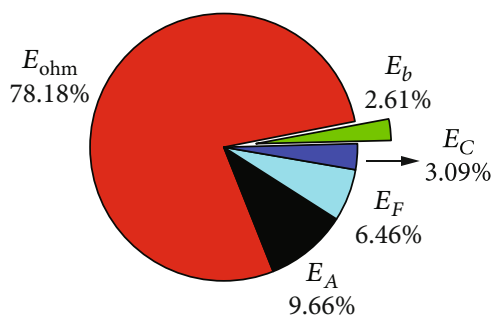

(d)

FIgURe 8: Dynamic behaviour of the battery system at maximum power consumption. The mission time is about $359 \mathrm{~h}$ : (a) SOC v. mission time; (b) power change from time $0 \mathrm{~h}$ to $15 \mathrm{~h}$; (c) power change from time $305 \mathrm{~h}$ to $320 \mathrm{~h}$; (d) energy ratio distribution.

\section{Results and Analysis}

As shown in Figure 7, to verify the HCPC model with variable voltage (AC mode in Figure 7) and code, a comparison was made with a research by Sánchez-Arriaga et al. [34] on the influence of different constant bias voltages $(0,20$, and $40 \mathrm{~V}$ in Figure 7). The deorbiting mission ranges from 800 to $500 \mathrm{~km}$ in altitude, and the dimensions of the tether are $3 \mathrm{~km}, 4 \mathrm{~cm}$, and $50 \mu \mathrm{m}$, respectively. The deorbiting durations under different inclinations are not significantly different from that of the constant voltages, and the growth pattern for the duration is also similar to that of the reference.

Table 1 shows the basic parameter settings. The resistivity and mass density are values representative of aluminium which is the preferred material used for such tethers at present. Other parameters may be changed based on this table in the following variable parameter research.

The accuracy of the result is affected by the time step length: a short time step can make the result closer to reality, but it requires more computing resources. In the simulation, an appropriate time step should be set as the maximum time step when using the Runge-Kutta-Fehlberg 4/5 method. Table 2 shows the total energy that can be harvested by the battery subsystem and the orbit transfer time for different time steps. Actually, the deviations are acceptable, given they are all less than $1 \%$. For higher resolution in describing SOC, $90 \mathrm{~s}$ is chosen for all calculations.
3.1. Dynamic Characteristic of the Battery Subsystem. For the battery subsystem is directly connected to the tether, it should work all the time to ensure the current loop on the tether and operation of the system throughout the mission. Figure 8 demonstrates the dynamic behaviour of the battery subsystem under the conditions set by the basic input parameters. The power consumption $P_{o}$ of the electrical appliances on board is set to be $39.7 \mathrm{~W}$ which is the maximum value that the battery subsystem can afford. The initial electric quantity is $10 \mathrm{~A} \bullet \mathrm{h}$ while the whole energy power consumed is about $350 \mathrm{~A} \bullet \mathrm{h}$. At the beginning of the mission, the plasma, in high orbit, cannot induce enough current in the tether, so the average power harvested by the battery subsystem (nearly $36 \mathrm{~W}$ ) is lower than the total power required. Due to the lack of energy, the battery begins to provide energy to the system, especially at night (in the shadow of the Earth). Although sometimes the power harvested exceeds the power demanded, the battery system mainly works in discharge mode. The SOC decreases until it reaches the maximum DOD at normalized time 0.41 . After that, the average power increases as the plasma density increases in low orbit. The battery system begins to work mainly in charging mode, and the SOC increases. In the last part of the mission, the power that can be harvested is much greater than the power consumption. The battery is full for most of the time, while the excess electrical energy has to be consumed in the protection circuit. In the mission, the tether is ohm-dominant so 


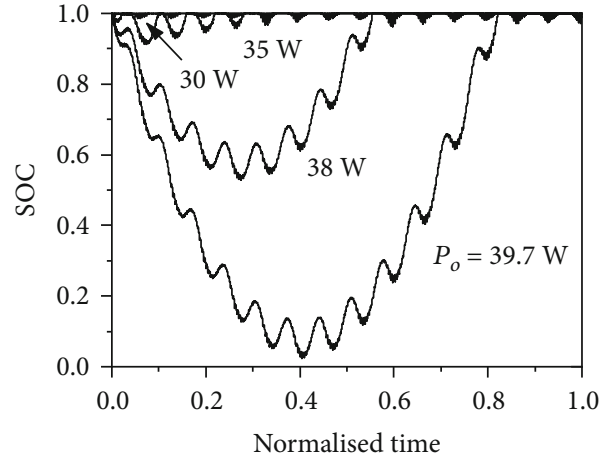

(a)

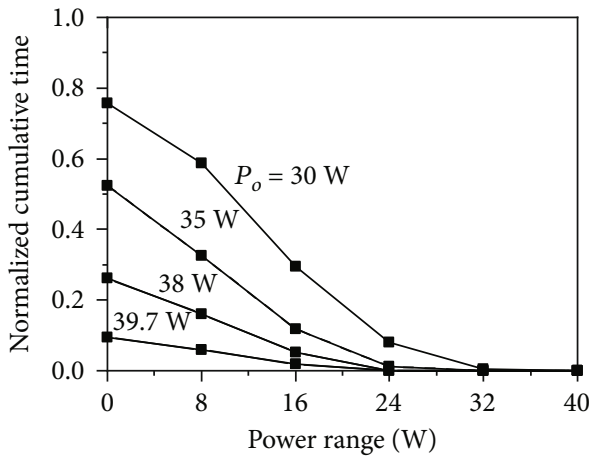

(b)

FIGURE 9: Working performance at different power consumptions: (a) SOC v. time; (b) waste power v. cumulative time; the abscissa plots the power range from $x$ to $40 \mathrm{~W}$.

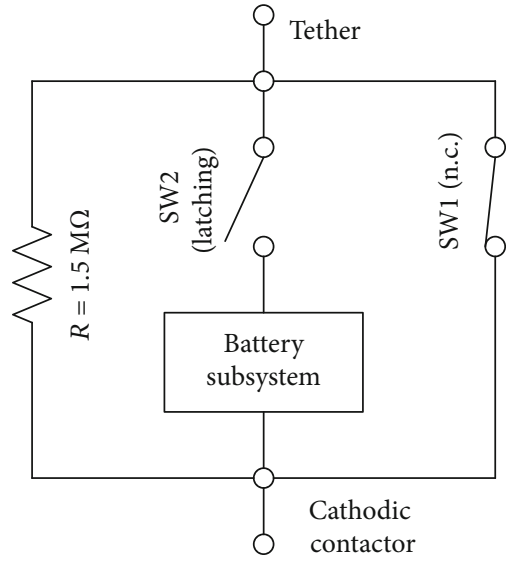

(a)

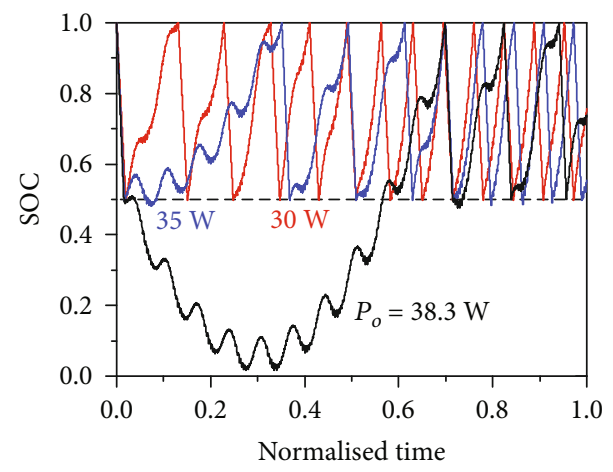

(b)

FIGURE 10: Strategy employed in ProSEDS: (a) simplified circuit; (b) SOC v. time.

that the energy dissipated on the tether $(78.18 \%)$ is much larger than that stored in the battery subsystem $(2.61 \%)$. The energy conversion efficiency (the ratio of $E_{b}$ to $\Delta E_{\text {orb }}$ ) is small, but the battery subsystem can provide the required power. For this application, the average harvested power, or maximum available power, is more important (these are $40.5 \mathrm{~W}$ and $39.7 \mathrm{~W}$, respectively). The effect of the atmospheric drag accounts for $6.46 \%$ of the orbital energy change such that it reduces the mission time.

The dynamic variation curve of the SOC of the battery subsystem looks like a "valley." It reaches the maximum DOD only once and does not have typical chargingdischarging cycles (it is charged and discharged to certain SOC values several times). As explained, the process can be divided into three periods of time: discharging phase I, charging phase II, and stable phase III. The initial stored electrical power in the battery is used to help pass through phase I which determines the success of the mission. If sufficient, the battery can enter its charging phase and then the stable phase which means that the mission can be completed. Generally, a well-designed battery subsystem will at least experience all of phase I and most of phase II which represents achievement of its maximum capability. The phrase "welldesigned" suggests that the battery characteristics $V_{b}$ and $Q_{\max }$ should match debris mitigation requirements (tether parameters, orbit parameters, and power consumption $P_{o}$ ); otherwise, it will be problematic with regard to the battery subsystem.

3.2. Differences with the Strategy Employed in ProSEDS. Regardless of the power consumption on board, the same bare electrodynamic tether system can complete the debris mitigation mission under different conditions, as long as the mission time can be accepted by the user; however, the flexibility of the battery subsystem is much lower than that of the tether. Figure 9(a) shows the working performance of the battery subsystem at different power consumption levels. A smaller power consumption leads to a low DOD. The duration of the discharging and charging regions decreases as the power consumption decreases as the difference between demand power and harvested energy is reduced. The behaviour of the stable region is opposite to that in regions I and II. The low power consumption means a large stable region; however, the presence of this stable region implies that the 


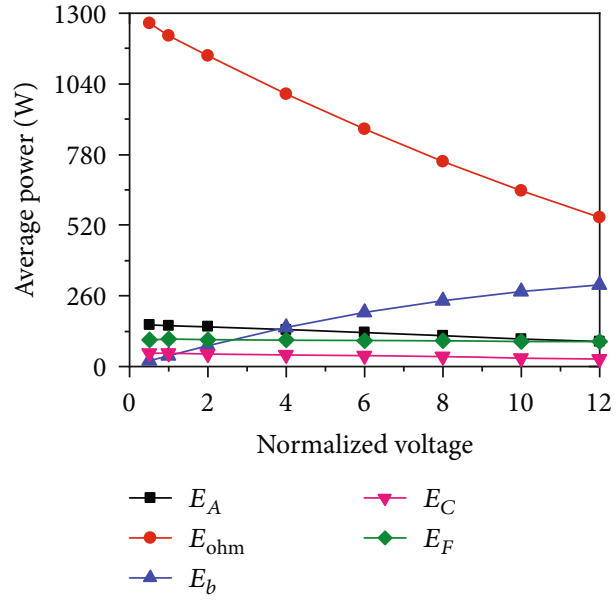

(a)

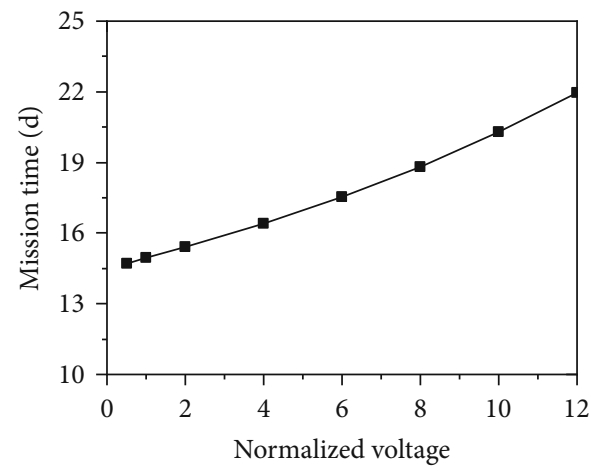

(c)

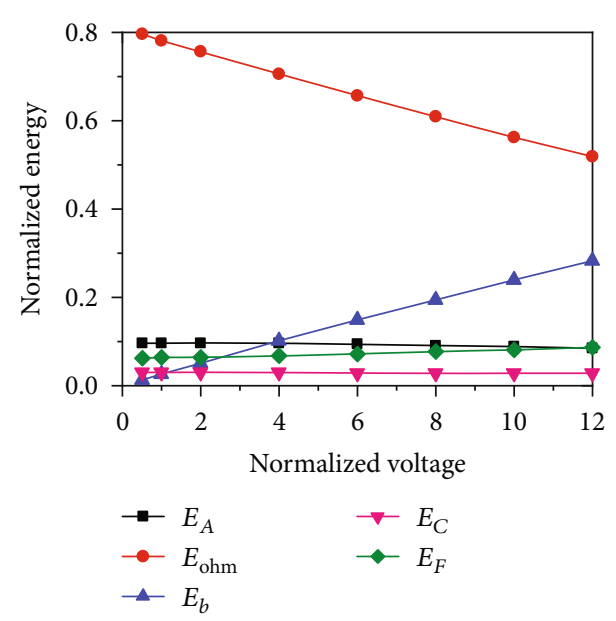

(b)

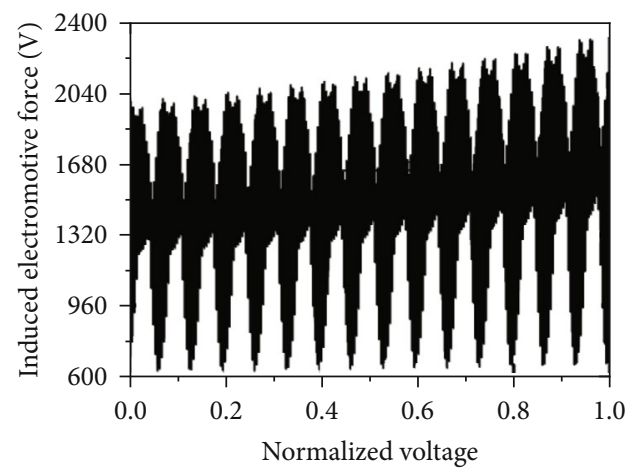

(d)

FIGURE 11: Power generation performance for different battery voltages: (a) average power v. $V_{b}$; (b) normalized energy v. $V_{b}$; (c) mission time v. $V_{b}$; (d) induced electromotive force v. mission time for the basic parameters.

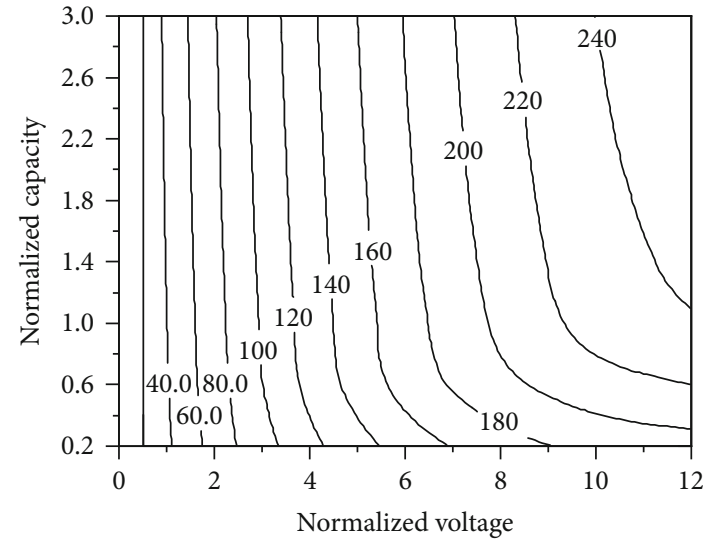

(a)

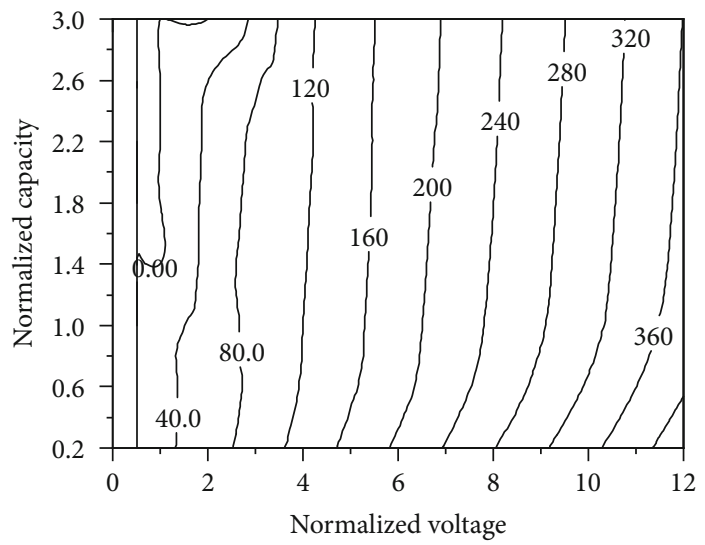

(b)

FIGURE 12: Battery performance for different battery parameters, and the capacity $(\mathrm{W} \cdot \mathrm{h})$ is normalized to $10 \times 41.9(\mathrm{~W} \cdot \mathrm{h}):(\mathrm{a}) \mathrm{maximum}$ available power; (b) maximum waste power.

energy harvested by the battery system is not all transferred to useful ends (battery module or electrical appliances): the longer the stable region, the worse the utilization rate. The ratios of useful energy (including consumed on board and stored in the battery) to total energy which has been har- vested by the battery are $0.738,0.860,0.934$, and 0.976 when the power consumption is $30 \mathrm{~W}, 35 \mathrm{~W}, 38 \mathrm{~W}$, and $39.7 \mathrm{~W}$, respectively. A large amount of energy should be dissipated in the protection circuit of the battery subsystem in the form of Joule heat, leading to a critical problem which has not been 
TABLE 3: Battery performance under different tether parameters.

\begin{tabular}{lccc}
\hline Condition 1: constant tether length $l$ & & & \\
Tether & & & \\
$\quad$ Width, $w(\mathrm{~cm})$ & 0.25 & 0.5 & 1 \\
$\quad$ Thickness, $h(\mu \mathrm{m})$ & 80 & 40 & 20 \\
Maximum available power $(\mathrm{W})$ & 35.3 & 39.7 & 42.4 \\
Deorbiting time (h) & 417.50 & 358.95 & 316.98 \\
Energy conversion efficiency (\%) & 2.82 & 2.61 & 2.31 \\
Maximum waste power (W) & 29.81 & 23.56 & 0 \\
\hline Condition 2: constant tether thickness $h$ & & \\
Tether & & & \\
$\quad$ Width, $w$ (cm) & 0.25 & 0.5 & 1 \\
$\quad$ Length, $l$ (km) & 20 & 10 & 5 \\
Maximum available power (W) & 23.6 & 39.7 & 54.4 \\
Deorbiting time (h) & 302.30 & 358.95 & 487.65 \\
Energy conversion efficiency (\%) & 1.21 & 2.61 & 5.50 \\
Maximum waste power (W) & 0 & 23.56 & 69.21 \\
\hline
\end{tabular}

TABLE 4: Battery performance for different debris masses.

\begin{tabular}{lccc}
\hline & \multicolumn{3}{c}{ Debris mass, $m_{d}(\mathrm{~kg})$} \\
& 750 & 1500 & 3000 \\
\hline Maximum available power (W) & 41.9 & 39.7 & 38.1 \\
Deorbiting time (h) & 182.44 & 358.9 & 705.6 \\
Energy conversion efficiency (\%) & 2.61 & 2.61 & 2.61 \\
Maximum waste power (W) & 0 & 23.56 & 28.33 \\
\hline
\end{tabular}

considered in previous energy analysis of tether systems. Figure 9(b) shows the cumulative time for a certain wasted power range in which the battery system will suffer. The value of waste power can be higher than $24 \mathrm{~W}$, and the cumulative time is considerable. To radiate the heat generated at such a high power and for a long period, a special structural design or electronic components are required. If not, the performance and lifetime of the battery subsystem will be impaired.

The battery subsystem is directly connected to the tether so that it cannot be cut-off to stop the energy harvesting in the stable region. This is the most basic difference when compared with the strategy employed in ProSEDS [23]. In ProSEDS, high-voltage relays were placed between the tether and the battery system and tether and contactor (Figure 10(a)). If necessary, the battery subsystem can be disconnected from the tether main circuit, and the current in the tether can flow through another line, directly reducing the heating power. Figure 10(b) illustrates the dynamic behaviour at constant power consumption under the hypothesis that the mode transition points are $\mathrm{SOC}=0.5$ and $\mathrm{SOC}=1$. The tether fails to complete the mission when $P_{o}$ is $39.7 \mathrm{~W}$ (the maximum power drops to $38.3 \mathrm{~W}$ ). When $P_{o}$ is large, the SOC change also looks like a "valley" at the beginning of the mission. In other situations, the battery works as normal. In these cases, the number of mode conversion is no more than 10 times. Mode conversion frequency mainly depends on the ratio of energy harvested to that consumed. The impressive advantage of this strategy is that the system can complete the mission with a wide range of power demand without heating problems. The concern is that the relays need extra energy and are potential failure points. By comparison, the advantage of our direct connection strategy is that a relatively stable voltage drop can be maintained along one end of the tether, avoiding potential dynamic oscillation due to change in current transient.

3.3. Battery Performance of Different Battery Parameters. As mentioned above, the design of the battery subsystem should match the mission parameters. The available power for electrical appliances is mainly determined by both $V_{b}$ and $Q_{\max }$. $V_{b}$ determines the maximum energy that the battery system can harvest, while $Q_{\max }$ determines the proportion of the energy which can be actually used. $V_{b}$ is more important as it is directly proportional to the power so that past research only focuses on it or the equivalent resistance. Figures 11(a) and 11(b) show the battery performance for different $V_{b}$ (normalized to the value in Table 1). The energy conversion efficiency for the battery subsystem is nearly linearly proportional to the battery voltage; however, the high battery voltage makes the voltage of the tether anode small (Figure 4), leading to a small tether current which further results in long mission time (shown in Figure 11(c)). Due to the decreasing tether current, the average power increases gradually to a maximum (about $260 \mathrm{~W}$ here) with the energy conversion efficiency rising to nearly $30 \%$. If the voltage increases further, the average power will instead decrease [24, 25], which was not calculated in our simulation. It is worth mentioning that there should be a limitation on the battery voltage selection. Once it exceeds the minimum of the induced electromotive force on tether, the whole system will repeatedly suffer no current situations. From Figure 11(d), the maximum proposed voltage is no more than $600 \mathrm{~V}$ (a normalized value of about 14).

By contrast, $Q_{\max }$ does not affect the amount of power generation: it determines the actual available power supply for the appliances, but its effect on available power is not as obvious as that of $V_{b}$. Despite this, it is necessary for power supply optimization. Figure 12 shows the maximum available power and waste power in different battery parameters. For a given power requirement (from those electrical appliances on board), several combinations of battery parameters can realize the demand: a larger battery voltage means less system mass; however, the maximum waste power on board limits such design; large battery capacity can reduce the waste power but that makes the system heavy and also directly decreases the economy (the ratio of battery capacity to total power consumed by on-board electrical appliances). Therefore, the trade-off between battery system mass and maximum waste power should be considered. From Figure 12, small battery voltage with sufficient capacity is preferred. For the conditions given in Table 1 , when $P_{o}$ is $40 \mathrm{~W}$, a battery voltage at 1 with a battery capacity of 1.4 is a better choice. Meanwhile, a higher output makes the DOD lower which means a lower task risk. If the power requirement is 
TABLE 5: Battery performance for different orbit parameters.

\begin{tabular}{lccccc}
\hline Variable & Value & $\begin{array}{c}\text { Maximum available power } \\
(\mathrm{W})\end{array}$ & $\begin{array}{c}\text { Mission time } \\
(\mathrm{h})\end{array}$ & $\begin{array}{c}\text { Energy conversion efficiency } \\
(\%)\end{array}$ & $\begin{array}{c}\text { Maximum waste power } \\
(\mathrm{W})\end{array}$ \\
\hline \multirow{2}{*}{ Initial semimajor axis } & 6800 & 44.7 & 235.32 & 1.74 & 0 \\
$(\mathrm{~km})$ & 6900 & 39.7 & 358.95 & 2.61 & 23.56 \\
& 7000 & 33.4 & 457.42 & 2.03 & 29.22 \\
\hline & 0 & 52.1 & 232.87 & 2.27 & 0 \\
Inclination $\left(^{\circ}\right)$ & 15 & 48.4 & 260.40 & 2.61 & 20.58 \\
& 30 & 39.7 & 358.95 & 3.18 & 23.56 \\
& 45 & 29.7 & 575.52 & 3.72 & 23.03 \\
\end{tabular}

too high $(>60 \mathrm{~W})$, optimization of the tether structure design is recommended.

\subsection{Energy Harvesting Performance of Different Input} Parameters. Table 3 lists battery performances under different tether parameters. The tether mass is set to be a constant. In condition 1, the changes in maximum available power, deorbiting time, and energy conversion efficiency are all about $10 \%$ when the tether cross-section perimeter is about $5 \mathrm{~mm}, 10 \mathrm{~mm}$, and $20 \mathrm{~mm}$. A large perimeter means a high tether current, which leads to a higher maximum power, less deorbiting time, and a lower energy efficiency. It is suggested to increase the perimeter to generate more available power with less heating. The changes in condition 2 suggest the opposite. A long tether leads to a higher thrust, although the current is reduced because of the perimeter. The first three indices in Table 3 can change by as much as $66 \%$, $40 \%$, and $100 \%$, respectively, when the tether length is $20 \mathrm{~km}, 10 \mathrm{~km}$, and $5 \mathrm{~km}$. This implies that a long tether design is more appropriate for missions with a low onboard power demand.

The debris mass (the mass of mother spacecraft) is changed to half or twice the basic value in Table 4. A smaller debris mass naturally means a shorter mission time which is proportional to the mass. The ability to harvest energy from the orbit is little affected by the debris mass because the current in, and force on, the tether changes little. The energy conversion efficiency of the battery system is nearly the same which means that the maximum available power should be almost the same, but as the burden of electrical power needed to make up the energy deficit is reduced by a shorter discharging section, the maximum available power is increased when the mass is reduced.

As shown in Table 5, the performance of the battery system changes regularly when the orbital parameters change. The low semimajor axis brings a higher plasma density, while the low inclination mainly means a higher plasma density with higher effective geomagnetic field intensity (the part which is perpendicular to the system velocity). When the semimajor axis and inclination are low, the current induced in the tether is large, so the maximum power is large, and the mission time is reduced. The ratio of maximum power to maximum waste power decreases as the semimajor axis and inclination increase: if the inclination is too high (greater than $60^{\circ}$ ), the maximum power drops to an extremely low value such that a battery system with a high battery voltage will be required.

\section{Conclusions}

(1) A numerical model is developed to calculate the power generation performance of a bare electrodynamic tether based on a Li-ion battery subsystem debris mitigation in space. The basic working performance, power generation strategy analysis, battery optimization, and applicability of the tether system are all discussed

(2) Four parameters can be used to describe the performance of the power generation: mission time, energy conversion efficiency, maximum available power, and maximum waste power. The first three are used to view the mitigation mission and the performance in power generation, while the last is employed to discuss the applicability of the battery system

(3) Generally, the SOC of the battery works as a "valley": this is unavoidable because of the large variation in space plasma parameters during orbit transfer. In the last part of the mission (in some cases), the power harvested will be much larger than that demanded. The excess energy should be dissipated for the battery subsystem cannot be cut off from the current loop, leading to a heating problem in the battery system. The extent of the problem is represented by maximum waste power. Extra control circuit which help the battery subsystem cut off from the tether can avoid the heating problem

(4) The battery voltage directly determines magnitude of the maximum power while the battery capacity affects the final available value and waste power. It is suggested to find a combination of a low battery voltage and a large battery capacity for the battery design to avoid the heating problem; however, this choice will lead to the mass of the battery system increasing so that the trade-off between mass and waste power should be considered 
(5) A wide tether can increase the maximum available power in an efficient manner, while a long, thin, tether is more advantageous for low power-requirement missions. The debris mass does not have a significant effect on battery performance except with regard to the required mission time. A high semimajor axis and a high inclination of orbit increase the mission time, and the maximum available power is lower therewith

\section{Nomenclature}

B: $\quad$ Geomagnetic field vector, $\mathrm{T}$

F: Perturbation force vector, $\mathrm{N}$

$\mathbf{F}_{\text {air }}$ : Atmospheric drag vector, $\mathrm{N}$

$\mathbf{F}_{\mathrm{EDT}}$ : Lorentz force vector, $\mathrm{N}$

L: $\quad$ Tether vector, $m$

r: $\quad$ Tether position vector, $m$

S: $\quad$ Tether windward side vector

v: Velocity vector of system mass centre, $\mathrm{m} / \mathrm{s}$

$A_{t}: \quad$ Tether cross-section area, $\mathrm{m}^{2}$

$e: \quad$ Electron charge, $\mathrm{C}$

$E_{A}: \quad$ Energy dissipated by tether anode, $\mathrm{J}$

$E_{b}$ : Energy harvested by battery system, J

$E_{C}$ : Energy dissipated by cathodic contactor, J

$E_{F}$ : Work done by atmospheric drag, J

$E_{m}:$ Induced electromotive force, $\mathrm{V} / \mathrm{m}$

$E_{\text {ohm }}$ : Energy dissipated by tether resistance, J

$E_{\text {orb }}:$ Orbital energy, J

$h$ : Geographic height, $\mathrm{km}$

I: $\quad$ Tether current, A

$I_{A}$ : Tether anode current, A

$I_{b}$ : Battery system current, A

$I_{\mathrm{bi}}$ : Battery module current, A

$I_{\mathrm{C}}$ : Cathodic contactor current, A

$l$ : $\quad$ Tether length, $\mathrm{m}$

$L_{0}$ : $\quad$ Distance from anode to a point where $V_{p}=V_{t}, \mathrm{~m}$

$m$ : Whole system mass, $\mathrm{kg}$

$m_{e}: \quad$ Electron mass, $\mathrm{kg}$

$m_{i}: \quad$ Ion mass, $\mathrm{kg}$

$N_{e}: \quad$ Electron density, $\mathrm{m}^{-3}$

$N_{i}: \quad$ Ion density, $\mathrm{m}^{-3}$

$p: \quad$ Tether cross-section perimeter, $\mathrm{m}$

$P_{b}$ : Battery system energy harvesting power, W

$P_{o}$ : $\quad$ Demand power on board, $\mathrm{W}$

$P_{w}: \quad$ Battery system waste power, $\mathrm{W}$

$Q_{\max }:$ Battery capacity, A $\bullet h$

$r$ : Magnitude of position vector, $\mathrm{m}$

$t: \quad$ Time, $s$

$v$ : Velocity of system mass centre, $\mathrm{m} / \mathrm{s}$

$V_{A}$ : Tether anode potential to plasma potential, $\mathrm{V}$

$V_{b}$ : Battery system voltage, $\mathrm{V}$

$V_{C}$ : Contactor bias voltage, $\mathrm{V}$

$V_{p}: \quad$ Plasma potential, $\mathrm{V}$

$V_{t}: \quad$ Tether potential, $\mathrm{V}$

$W$ : $\quad$ Work done by perturbation force, $\mathrm{J}$

$\mu: \quad$ Gravitational parameter, $\mathrm{m}^{3} / \mathrm{s}^{2}$

$\rho$ : Atmospheric mass density, $\mathrm{kg} / \mathrm{m}^{3}$

$\sigma: \quad$ Tether conductivity, $\mathrm{S} / \mathrm{m}$.

\section{Data Availability}

The data used to support the findings of this study are available from the corresponding author upon request.

\section{Conflicts of Interest}

The authors declare that there is no conflict of interest regarding the publication of this paper.

\section{Acknowledgments}

This work was supported by the advanced research project in manned space (grant number 0130).

\section{References}

[1] K. Lemmer, "Propulsion for CubeSats," Acta Astronautica, vol. 134, pp. 231-243, 2017.

[2] J. R. Sanmartín, M. Martinez-Sanchez, and E. Ahedo, "Bare wire anodes for electrodynamic tethers," Journal of Propulsion and Power, vol. 9, no. 3, pp. 353-360, 1993.

[3] J. R. Sanmartín, R. D. Estes, E. C. Lorenzini, and S. A. Elaskar, "Efficiency of electrodynamic tether thrusters," Journal of Spacecraft and Rockets, vol. 43, no. 3, pp. 659-666, 2006.

[4] J. R. Sanmartín, M. Charro, X. Chen et al., "A universal system to de-orbit satellites at end of life," Journal of Space Technology \& Science, vol. 26, 2012.

[5] I. C. Bell III, B. E. Gilchrist, J. K. McTernan, and S. G. Bilén, "Investigating miniaturized electrodynamic tethers for picosatellites and femtosatellites," Journal of Spacecraft and Rockets, vol. 54, no. 1, pp. 55-66, 2017.

[6] C. Pardini, T. Hanada, and P. H. Krisko, "Benefits and risks of using electrodynamic tethers to de-orbit spacecraft," Acta Astronautica, vol. 64, no. 5-6, pp. 571-588, 2009.

[7] H. Hakima and M. R. Emami, "Assessment of active methods for removal of LEO debris," Acta Astronautica, vol. 144, pp. 225-243, 2018.

[8] J. R. Sanmartín, A. Sánchez-Torres, S. B. Khan, G. SánchezArriaga, and M. Charro, "Optimum sizing of bare-tape tethers for de-orbiting satellites at end of mission," Advances in Space Research, vol. 56, no. 7, pp. 1485-1492, 2015.

[9] K. Makihara and S. Kondo, "Structural evaluation for electrodynamic tape tethers against hypervelocity space debris impacts," Journal of Spacecraft and Rockets, vol. 55, no. 2, pp. 462-472, 2018.

[10] H. Tahara, H. Nishio, and T. Onishi, "Basic study of electron collection by a bare-tether satellite," Vacuum, vol. 73, no. 34, pp. 455-460, 2004.

[11] G. Li, Z. H. Zhu, J. Cain, F. Newland, and A. Czekanski, "Libration control of bare electrodynamic tethers considering elastic-thermal-electrical coupling," Journal of Guidance, Control, and Dynamics, vol. 39, no. 3, pp. 642-654, 2016.

[12] A. Y. Aleksandrov and A. A. Tikhonov, "Asymptotic stability of a satellite with electrodynamic attitude control in the orbital frame," Acta Astronautica, vol. 139, pp. 122-129, 2019.

[13] Y. Yang and H. Cai, "Libration dynamics of electrodynamic tether system for 13 degrees International Geomagnetic Reference Field," Acta Astronautica, vol. 151, pp. 182-205, 2018. 
[14] S. B. Khan and J. R. Sanmartín, "Analysis of tape tether survival in LEO against orbital debris," Advances in Space Research, vol. 53, no. 9, pp. 1370-1376, 2014.

[15] K. Makihara and R. Takahashi, "Survivability evaluation of electrodynamic tethers considering dynamic fracture in space-debris impact," Journal of Spacecraft and Rockets, vol. 53, no. 1, pp. 209-216, 2016.

[16] C. Bombardelli, D. Zanutto, and E. Lorenzini, "Deorbiting performance of bare electrodynamic tethers in inclined orbits," Journal of Guidance, Control, and Dynamics, vol. 36, no. 5, pp. 1550-1556, 2013.

[17] G. Li, Z. H. Zhu, S. Ruel, and S. A. Meguid, "Multiphysics elastodynamic finite element analysis of space debris deorbit stability and efficiency by electrodynamic tethers," Acta Astronautica, vol. 137, pp. 320-333, 2017.

[18] C. Bombardelli, J. Pelaez, and M. Sanjurjo, "Asymptotic solution for the current profile of passive bare electrodynamic tethers," Journal of Propulsion and Power, vol. 26, no. 6, pp. 1291-1304, 2010.

[19] C. Bombardelli, "Power density of a bare electrodynamic tether generator," Journal of Propulsion and Power, vol. 28, no. 3, pp. 664-668, 2012.

[20] J. R. Sanmartín, E. C. Lorenzini, and M. Martinez-Sanchez, "Electrodynamic tether applications and constraints," Journal of Spacecraft and Rockets, vol. 47, no. 3, pp. 442-456, 2010.

[21] C. Bombardelli, E. C. Lorenzini, and J. R. Sanmartín, "Jupiter power generation with electrodynamic tethers at constant orbital energy," Journal of Propulsion and Power, vol. 25, no. 2, pp. 415-423, 2009.

[22] S. Bilen, J. McTernan, B. Gilchrist, I. Bell, N. Voronka, and R. Hoyt, "Electrodynamic tethers for energy harvesting and propulsion on space platforms," in AIAA SPACE 2010 Conference \& Exposition, pp. 2010-8844, Anaheim, CA, USA, 2010.

[23] L. Johnson, B. Gilchrist, E. Lorenzini, N. Stone, and K. Wright, "Propulsive small expendable deployer system (ProSEDS) experiment: mission overview and status," in 39th AIAA/ASME/SAE/ASEE Joint Propulsion Conference and Exhibit, pp. 2003-5094, Huntsville, AL, USA, 2003.

[24] J. McTernan, B. Gilchrist, S. Bilen, R. Hoyt, N. Voronka, and I. Bell, "Development of a modeling capability for energy harvesting modules in electrodynamic tether systems," in AIAA SPACE 2011 Conference \& Exposition, pp. 2011-7323, Long Beach, CA, USA, 2011.

[25] R. P. Hoyt, "Propulsion and power harvesting performance of electrodynamic tethers," in AIAA SPACE 2011 Conference \& Exposition, pp. 2011-7321, Long Beach, CA, USA, 2011.

[26] M. Sanjurjo-Rivo and J. Peláez, "Energy analysis of bare electrodynamic tethers," Journal of Propulsion and Power, vol. 27, no. 1, pp. 246-256, 2011.

[27] J. K. Mcternan and S. G. Bilén, "Embodied energy repurposing via energy-harvesting electrodynamic tethers," Journal of Spacecraft and Rockets, vol. 54, no. 4, pp. 789-795, 2017.

[28] H. D. Curtis, Orbital Mechanics for Engineering Students, Butterworth-Heinemann, 3rd edition, 2014.

[29] D. Bilitza and B. W. Reinisch, "International reference ionosphere 2007: improvements and new parameters," Advances in Space Research, vol. 42, no. 4, pp. 599-609, 2008.

[30] A. Chulliat, W. Brown, P. Alken et al., "The US/UK world magnetic model for 2015-2020: technical report," National Oceanic and Atmospheric Administration (NOAA), 2015.
[31] J. M. Picone, A. E. Hedin, D. P. Drob, and A. C. Aikin, "NRLMSISE-00 empirical model of the atmosphere: statistical comparisons and scientific issues," Journal of Geophysical Research, vol. 107, no. A12, pp. SIA 15-1-SIA 15-16, 2002.

[32] E. Ahedo and J. R. Sanmartín, "Analysis of bare-tether systems for deorbiting low-earth-orbit satellites," Journal of Spacecraft and Rockets, vol. 39, no. 2, pp. 198-205, 2002.

[33] K. Xie, R. A. Martinez, and J. D. Williams, "Current-voltage characteristics of a cathodic plasma contactor with discharge chamber for application in electrodynamic tether propulsion," Journal of Physics D: Applied Physics, vol. 47, no. 15, article 155501, 2014

[34] G. Sánchez-Arriaga, C. Bombardelli, and X. Chen, "Impact of nonideal effects on bare electrodynamic tether performance," Journal of Propulsion and Power, vol. 31, no. 3, pp. 951-955, 2015. 